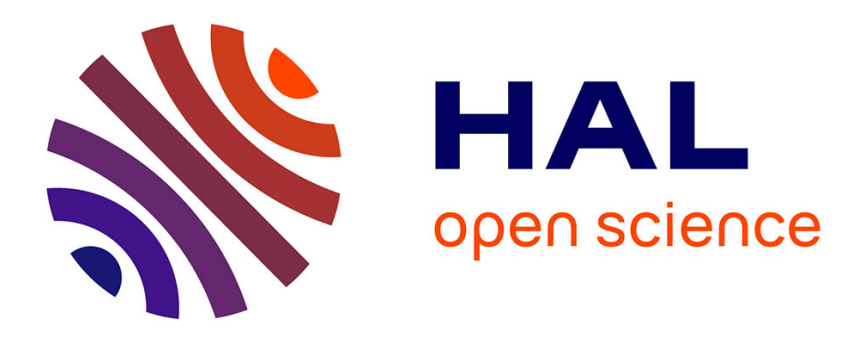

\title{
Etude expérimentale d'une décharge d'arc électrique contaminée par des vapeurs d'isolants
}

\author{
B. Cheminat, P. Andanson
}

\section{To cite this version:}

B. Cheminat, P. Andanson. Etude expérimentale d'une décharge d'arc électrique contaminée par des vapeurs d'isolants. Revue de Physique Appliquée, 1986, 21 (2), pp.187-193. 10.1051/rphysap:01986002102018700 . jpa-00245424

\section{HAL Id: jpa-00245424 https://hal.science/jpa-00245424}

Submitted on 1 Jan 1986

HAL is a multi-disciplinary open access archive for the deposit and dissemination of scientific research documents, whether they are published or not. The documents may come from teaching and research institutions in France or abroad, or from public or private research centers.
L'archive ouverte pluridisciplinaire HAL, est destinée au dépôt et à la diffusion de documents scientifiques de niveau recherche, publiés ou non, émanant des établissements d'enseignement et de recherche français ou étrangers, des laboratoires publics ou privés. 


\title{
Etude expérimentale d'une décharge d'arc électrique contaminée par des vapeurs d'isolants
}

\author{
B. Cheminat et P. Andanson \\ Laboratoire de Physique des Matériaux, Université de Clermont-Ferrand, BP 45, 63170 Aubière, France
}

(Reçu le 10 mai 1985, révisé le 10 octobre, accepté le 12 novembre 1985)

\begin{abstract}
Résumé. - Une étude expérimentale a permis de montrer que la présence de vapeurs issues de certains isolants industriels entraîne une augmentation de la température, du champ électrique et de la conductivité thermique de la colonne d'un arc électrique ainsi qu'une diminution du temps d'extinction de l'arc. Les mesures sont faites par spectrométrie et par une méthode de sondes.
\end{abstract}

\begin{abstract}
Through an experimental investigation, it was shown that vapours originating from some industrial insulators cause an increase in the temperature, electric field and thermal conductivity of the column of an electric arc as well as a shortening of the arc extinction time. The measurements were carried out spectrometrically and using a probing method.
\end{abstract}

\section{Introduction.}

La miniaturisation des disjoncteurs, par ailleurs destinés à des puissances de plus en plus grandes, conduit à des densités d'énergie très importantes. Les chambres de coupure de ces appareils comportent généralement des pièces constituées d'isolant. Ce matériau peut, dans certains cas, être volatilisé dans la colonne lors du soufflage de l'arc. Or il est évident que des paramètres comme le temps d'extinction ou la probabilité de réamorçage dépendent en grande partie des propriétés physiques du plasma. Le but de l'étude entreprise est de mettre en évidence l'influence de vapeurs issues de certains isolants industriels sur les caractéristiques de la colonne d'arc. La spectrométrie constitue pour cette analyse un moyen de diagnostic privilégié.

Une première étude faite sur un plasma d'air dans lequel étaient vaporisés des isolants à base d'amiante et de ciment a montré la grande complexité des plasmas engendrés. Si certains résultats concernant la détection des raies ou les mesures du champ électrique ont pu être obtenus, leur exploitation s'est révélée très délicate. Aussi dans une première phase de recherche, cette étude a été reprise en utilisant l'argon comme gaz plasmagène. L'utilisation d'un gaz monoatomique facilite en effet l'exploitation des résultats spectroscopiques. L'étude qui va être présentée concerne un plasma d'argon contaminé par les vapeurs issues des isolants industriels suivants : amiante-ciment, tissu de fibre de verre imprégné de ciment, mélamine.

La composition de ces isolants est complexe. Elle a été déterminée à partir des indications du fabricant et des valeurs données par certains auteurs $[1,2]$.

La température, la densité électronique, le champ électrique et le temps d'extinction de l'arc ont été mesurés à l'aide d'un dispositif expérimental et de méthodes qui vont être présentés.

\section{Dispositif expérimental et méthodes de mesure.}

Le diagnostic du plasma [3] est fait à partir de deux méthodes différentes :

- la spectrométrie pour la détermination de la température, de la densité électronique et du temps d'extinction,

- l'utilisation de sondes pour évaluer le champ électrique.

Le dispositif expérimental est composé d' :

- un ensemble de production de l'arc,

- un dispositif de mesures spectrométriques,

- un système de mesure du champ électrique,

- un système de coupure de l'arc.

2.1 L'ARC. - Les mesures spectrométriques nécessitent une bonne stabilité de la colonne d'arc. Ce dernier est stabilisé par parois dans une chambre 
à arc de type Maecker dont une vue en coupe est représentée par la figure 1.

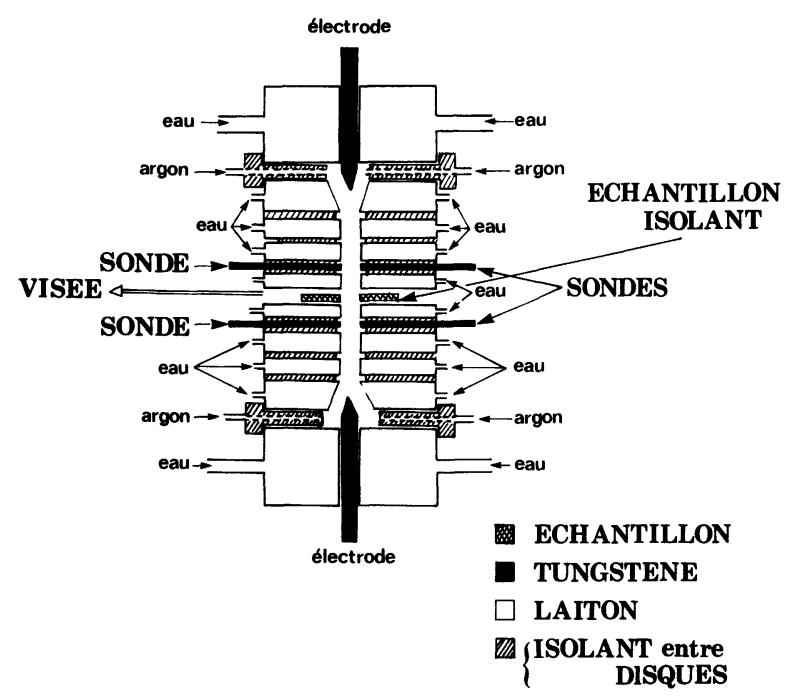

Fig. 1. - Vue en coupe de la chambre à arc.

[Cross section of the arc chamber.]

Cette chambre est composée de plusieurs coupelles en laiton, creuses, refroidies par eau et isolées les unes des autres.

Les électrodes sont en tungstène, cylindriques (diamètre $6 \mathrm{~mm}$ ) pointues (angle du cône $30^{\circ}$ ). Elles sont distantes de $4 \mathrm{~cm}$ et fortement refroidies par eau.

Le diamètre du canal de décharge est $6 \mathrm{~mm}$.

A chacune des extrémités de la chambre, un anneau en nylon assure l'isolation et l'arrivée d'argon. Le gaz est introduit tangentiellement à la colonne d'arc par deux orifices de diamètre $1,2 \mathrm{~mm}$ qui sont diamétralement opposés. Le débit d'argon est de 1,5 1/min.

La fenêtre de visée est située dans le plan médian de la colonne entre 2 coupelles de refroidissement.

Deux fentes sont disposées autour du canal de décharge (Fig. 1) afin de pouvoir amener les échantillons d'isolants à proximité de l'arc dans le but de les vaporiser. Le barreau d'isolant $(1 \times 10 \times 100 \mathrm{~mm})$ est introduit tangentiellement à la colonne d'arc à l'aide d'un dispositif qui l'entraîne avec une vitesse constante dans la chambre à arc. La vitesse de déplacement de l'échantillon est de $0,5 \mathrm{~mm} / \mathrm{s}$. Elle a été choisie de manière à assurer la meilleure stabilité du plasma. La stabilité de ce type de décharge reste néanmoins difficile à obtenir, la vaporisation du matériau n'étant jamais très régulière.

Les mesures ont été faites pour des intensités de courant d'arc comprises entre 20 et 50 A. Pour des valeurs supérieures, la vaporisation de l'échantillon devient trop rapide et irrégulière pour que des mesures soient possibles.

2.2 LE DISPOSITIF DE MESURES SPECTROMÉTRIQUES. Le montage est composé d'un monochromateur Très
Haute Résolution dont les caractéristiques sont les suivantes :

- type : Czerny-Turner

- focale : $1,5 \mathrm{~m}$

- réseau : $2400 \mathrm{t} / \mathrm{mm}$ (domaine spectral $200 \mathrm{~nm}<$

$\lambda<750 \mathrm{~nm}$ blazé à $550 \mathrm{~nm}$ )

- pouvoir théorique de résolution $: \frac{\lambda}{\Delta \lambda}=150000$ en simple passage.

Il est associé à un montage optique qui permet de former l'image de l'arc sur la fente d'entrée du monochromateur avec un grandissement de 2 . Le capteur est un photomultiplicateur à cathode frontale (Hamamatsu R1104) dont la courbe de réponse est pratiquement plate de 400 à $800 \mathrm{~nm}$. Il est relié à un amplificateur. Afin d'éviter les signaux parasites induits dans les câbles de raccordement, l'amplificateur est fixé contre le support du tube PM. Il comporte deux amplificateurs opérationnels. Le premier est utilisé en convertisseur courant-tension. Cette disposition permet de rendre la tension d'anode du PM indépendante du courant qu'il débite. Le réglage du gain, variable de 1 à 200, est obtenu par commutation de résistances (précision $1 \%$ ) au moyen de roues codeuses. Un potentiomètre permet de compenser le courant de repos du PM. La sortie de l'amplificateur est reliée à une table traçante.

La nécessité de réaliser un nombre important de mesures dans un temps raisonnable a conduit à piloter le dispositif spectrométrique par un microprocesseur (Motorola M6800) qui doit effectuer les commandes suivantes :

- la lecture des paramètres indispensables à l'exécution du programme (entrés au clavier par l'opérateur). Ce sont :

- la longueur d'onde de destination,

- la longueur d'onde initiale,

- le pas de la mesure (nul ou multiple de $2 \times$ $10^{-13} \mathrm{~m}$ ),

- l'origine (axe ou bord de la décharge) et le pas de déplacement latéral de la chambre à arc (réglage de 0 à $0,99 \mathrm{~mm}$ par pas de $0,01 \mathrm{~mm}$ ).

- La commande des moteurs entrainant la rotation du réseau :

- le moteur alternatif $(330 \mathrm{~nm} / \mathrm{min})$,

- le moteur pas à pas dont la vitesse de rotation peut varier de 0,005 à $5 \mathrm{~nm} / \mathrm{min}$.

- La commande du moteur pas à pas entraînant la chambre à arc dans son déplacement latéral avec une vitesse linéaire de $10^{-3} \mathrm{~ms}^{-1}$. Le mouvement de ce moteur est couplé à la rotation d'un potentiomètre dont la tension de sortie est appliquée à la voie $\mathrm{X}$ de la table traçante.

Un codeur optique d'impulsions (tous les $2 \times$ $10^{-12} \mathrm{~m}$ ) permet de vérifier le positionnement du réseau et sa vitesse de rotation.

$\mathrm{Ce}$ dispositif permet toutes les mesures nécessaires 
au diagnostic spectrométrique du plasma et permet ainsi de déterminer la température, la densité électronique et le temps d'extinction de l'arc.

La température. - La température est déterminée à partir de l'intensité absolue de la raie 415,8 nm de l'atome d'argon neutre dont les caractéristiques sont bien connues. Cette méthode est fréquemment utilisée pour connaître la température d'un plasma d'arc électrique.

Cette mesure nécessite l'inversion de l'équation intégrale d'Abel qui permet de calculer le coefficient d'émissivité locale $\varepsilon(r, \lambda)$ à partir de la valeur de l'énergie $I(x, \lambda)$ émise par le volume le long d'une corde (Fig. 2). La méthode utilisée est décrite dans l'article de Andanson et al. [4]. L'échantillonnage de la courbe $I(x, \lambda)$ conduit à 40 ou 50 points selon les courbes. L'interpolation est faite en utilisant des fonctions splines d'interpolation. L'intégrale est alors calculée exactement.

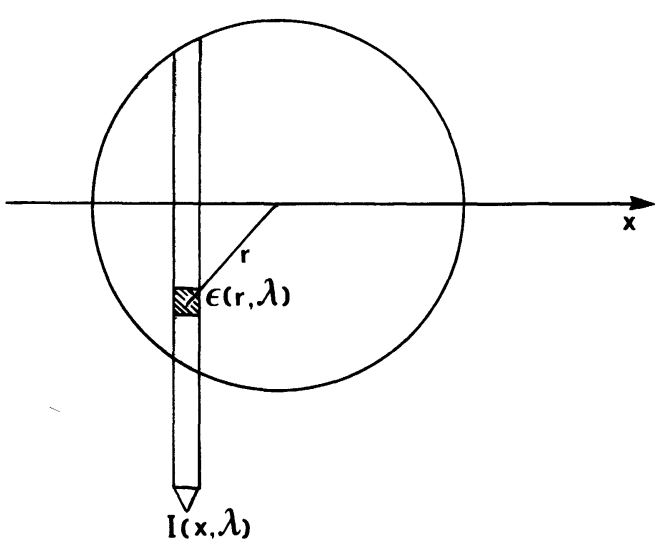

Fig. 2. - Vue en coupe de la colonne d'arc au niveau de l'axe de visée.

[Cross section of the arc column at the level of the axis of sight.]

La densité électronique. - La vaporisation de l'isolant dans le plasma conduit à la présence dans la colonne de 1 à $2 \%$ d'hydrogène. La mesure de la largeur à mi-hauteur de la raie $\mathrm{H}_{\beta}$ émise par l'atome neutre d'hydrogène permet de déterminer la valeur de la densité électronique [5].

La procédure à suivre est la suivante : on relève, pour une intensité de courant d'arc donnée, les profils de la raie $\mathrm{H}_{\beta}$ pour différentes valeurs de $x$ (40 valeurs). Ces courbes $I(\lambda)_{x}$ sont échantillonnées (33 points inégalement répartis en fonction de la longueur d'onde). La répartition des points tient compte de la forme du profil de raie. On obtient ainsi à partir des courbes $I(\lambda)_{x}$ les courbes $I(x)_{\lambda}$. L'inversion d'Abel conduit aux courbes $I(r)_{\lambda}$. Les profils $I(\lambda)_{r}$ peuvent alors être reconstitués. La largeur à mi-hauteur est mesurée. Il est nécessaire de faire un grand nombre de mesure et le temps d'acquisition des données est très long (45 $\mathrm{min})$. La stabilité des plasmas d'argon contaminés par des vapeurs d'isolants est difficile à obtenir et le temps d'acquisition de données doit être limité au maximum ( $<5 \mathrm{~min}$ ). La méthode employée est la suivante :

Pour 3 valeurs de l'intensité de courant d'arc la procédure précédemment décrite a été utilisée pour déterminer la densité électronique $N_{\mathrm{e}}$ d'un plasma d'argon contenant $2 \%$ d'hydrogène. Les valeurs de $N_{\mathrm{e}}$ obtenues ont été comparées à celles obtenues directement à partir des profils $I(\lambda)_{x}$ n'ayant pas été inversés. L'écart est toujours d'environ $30 \%$ (entre 27 et $34 \%$ ). Il faut majorer la valeur obtenue sans inversion d'Abel. Ce facteur de correction a été utilisé pour des plasmas d'argon contaminés par des vapeurs d'isolants. L'erreur faite en remplaçant l'inversion des profils par l'utilisation d'un facteur correctif reste dans tous les cas très inférieure à celle liée à l'utilisation de cette méthode qui est généralement estimée à $20 \%$ [5]. Les mesures sont alors effectuées en un temps compatible avec les impératifs expérimentaux.

Le temps d'extinction de l'arc. - Le temps $t_{\mathrm{c}}$ d'extinction de l'arc est celui au bout duquel l'intensité de la raie 415,8 $\mathrm{nm}$ de l'argon neutre a diminué de $90 \%$ à partir de l'instant de court-circuit de l'arc.

2.3 LE SYSTÈME DE COUPURE DE L'ARC. - Un thyristor rapide à gachette amplificatrice est monté en parallèle aux bornes de l'arc. Le temps d'amorçage du thyristor est de $2 \mu$ s.

Le temps de réponse du photomultiplicateur est de 3 ñs. Le montage optique est celui présenté précédemment. Le signal, prélevé directement à la sortie du PM, ainsi que la tension aux bornes de l'arc sont enregistrés à l'aide d'un dispositif d'acquisition de phénomènes transitoires. Ce système est un DataLab DL2800. L'échantillonnage est fait à une fréquence de $200 \mathrm{kHz}$. Dans chaque voie d'entrée, on peut stocker 4096 points codés sur 10 bits. Les informations ainsi mémorisées, peuvent être restituées soit sur un oscilloscope, soit sur une table traçante, soit sur une imprimante par l'intermédiaire d'un calculateur.

La constante de temps de l'appareillage est de l'ordre de $10 \mu \mathrm{s}$. Elle a été mesurée en remplaçant, pour un point de fonctionnement donné ( $\left.U_{\text {arc }}, I_{\text {arc }}\right)$, l'arc par une résistance pure de valeur $U_{\text {arc }} / I_{\text {arc }}$. Cette valeur est faible devant celle des temps d'extinction mesurés (120 à $150 \mu s)$.

\subsection{LE SYSTÈME DE MESURE DU CHAMP ÉLECTRIQUE. -} Il est généralement admis, par la plupart des auteurs, que dans la colonne d'un arc stabilisé, les surfaces équipotentielles, loin des zones de chutes anodique et cathodique, peuvent être représentées par le schéma de la figure 3.

La mesure du potentiel flottant entre deux points le long de la colonne permet de déterminer la valeur du champ électrique si on suppose ce dernier constant 


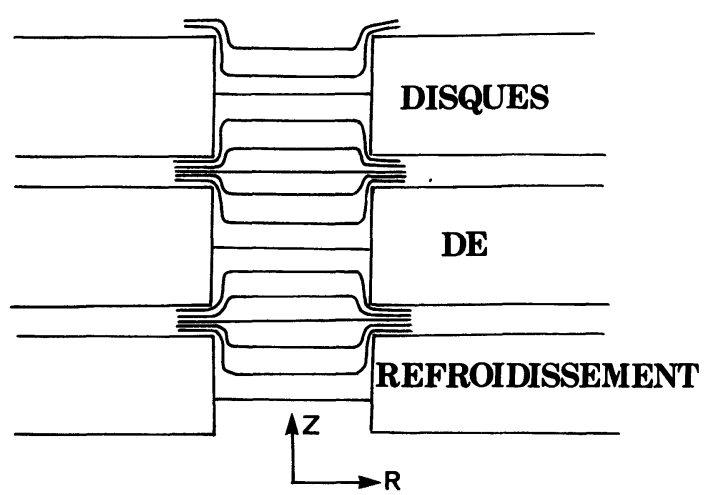

Fig. 3. - Distribution schématique des équipotentielles dans la colonne d'un arc stabilisé.

[Diagrammatic distribution of the equipotential curves in the column of a stabilized arc.]

entre ces deux points. Il faut mesurer la tension entre 2 sondes distantes de $l_{\mathrm{s}}$ et placées perpendiculairement à l'axe de la décharge.

Trois types de sondes sont possibles :

- les disques de refroidissement,

- les sondes fixes placées dans l'isolant entre les disques,

- les sondes mobiles pouvant être immergées dans le plasma.

Ces trois types de sondes ont été utilisés dans le cas d'un plasma d'argon exempt de toute contamination et très stable. Les résultats obtenus sont similaires [3]. Il faut noter que l'immersion dans la colonne d'arc de sondes mobiles provoque une perturbation qui peut être importante. Par ailleurs, les coupelles de refroidissement représentent pour le plasma des surfaces équipotentielles. Des essais ont été faits avec des coupelles d'épaisseurs différentes $(3,5,10 \mathrm{~mm})$. Aucune différence appréciable n'a été notée au cours des mesures. Les sondes utilisées pour la mesure du potentiel sont en aluminium, plates, rectangulaires, de $0,7 \mathrm{~mm}$ d'épaisseur et de $10 \mathrm{~mm}$ de largeur. Isolées avec des feuilles de mica, elles sont placées au bord de l'arc, de part et d'autre de la colonne, entre deux coupelles de refroidissement (Fig. 1). Les deux paires de sondes conduisent à des valeurs indépendantes.

L'évolution du champ électrique en fonction $d u$ temps est représenté par la courbe de la figure 4 . Cette courbe présente cinq parties :

0) en l'absence d'isolant, le champ électrique est celui d'un plasma d'argon pur ;

1) l'isolant arrive à proximité de la décharge et met un certain temps pour se vaporiser. On constate un faible abaissement du signal;

2) l'isolant est vaporisé par l'arc. Le champ électrique croît jusqu'à atteindre un maximum;

3) le signal reste maximum tant que l'isolant avance;

4) l'isolant est immobilisé, le champ électrique

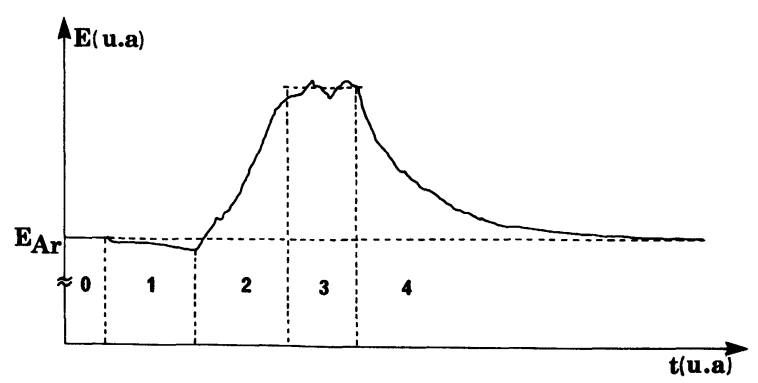

Fig. 4. - Evolution du champ électrique dans le temps. [Evolution of the electric field against time.]

décroît et reprend sa valeur initiale au bout de $25 \mathrm{~s}$ environ.

La décharge, très stable lorsque le plasma est exempt de toute contamination, présente, en présence de vapeurs d'isolants, une instabilité qui se traduit par des fluctuations de la tension d'arc ou de celle mesurée aux bornes des sondes. Pour des intensités de courant d'arc comprises entre 20 et $50 \mathrm{~A}$ les variations de tension entre les sondes sont de l'ordre de $10 \%$. Des variations de l'ordre de 1 à $2 \mathrm{~V}$ se produisent alors également sur la tension d'arc (40 V).

\section{Résultats et analyse.}

3.1 REChERCHE DE RAIES. - Comme le laisse prévoir la composition des isolants, le spectre émis se révèle très complexe. Les raies sont nombreuses, très proches et se superposent. Certaines raies de l'argon et de l'hydrogène $\left(\mathrm{H}_{\alpha} \mathrm{H}_{\beta}\right)$ peuvent être identifiées avec certitude; elles ont été utilisées pour l'analyse spectrométrique.

L'observation des premières courbes obtenues sur la table traçante permet une analyse préliminaire. Ces courbes (Fig. 5) représentent la répartition en fonction de $x$ de l'intensité totale de certaines raies et $\mathrm{du}$ fond continu correspondant. L'intensité de courant d'arc est de $30 \mathrm{~A}$. La visée est faite perpendiculairement aux barreaux d'isolant (Fig. 1).

Les courbes de la figure 5a montrent l'évolution de la raie $415,8 \mathrm{~nm}$ de l'atome d'argon en présence et en l'absence de vapeurs issues d'un tissu de fibre de verre et de ciment dans le plasma.

Les courbes de la figure $5 \mathrm{~b}$ montrent la répartition de l'intensité totale de la raie $415,8 \mathrm{~nm}$ de l'atome d'argon en présence et en l'absence de vapeurs issues d'amiante-ciment ainsi que l'évolution de la raie $656,2 \mathrm{~nm}\left(\mathrm{H}_{\alpha}\right)$ de l'atome d'hydrogène.

On peut en déduire que :

- l'isolant est présent jusqu'au coeur de l'arc.

- la présence de l'isolant diminue le diamètre optique de la colonne;

- la décharge présente une symétrie suivant l'axe $x$.

Ce dernier point suscite quelques remarques concernant l'hypothèse d'une géométrie de révolution 


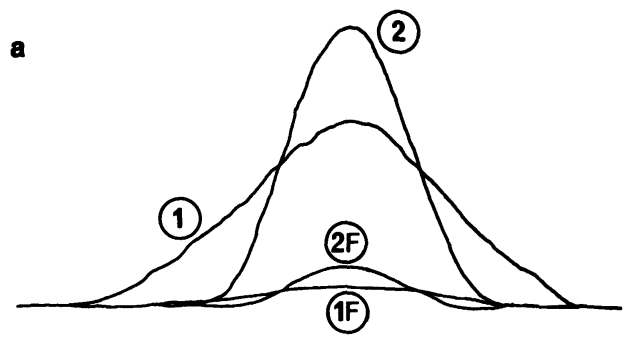

Raie $415,8 \mathrm{~nm}$ de l'atome d'argon.

(1) plasma d'argon pur

(2) plasma d'argon + amiante-ciment.

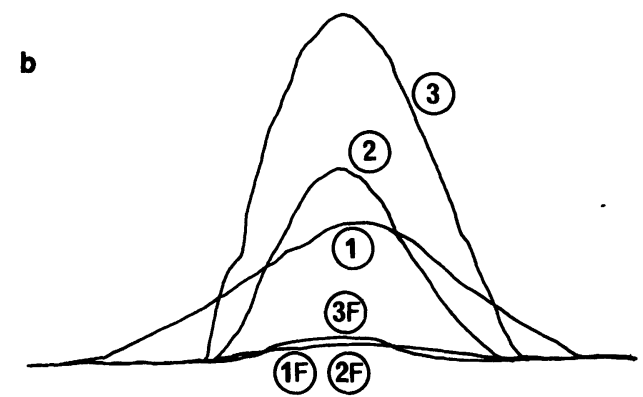

Raie 415,8 nm de l'argon.

(1) plasma d'argon pur

(2) plasma argon + amiante-ciment

(3) raie $656,2 \mathrm{~nm}\left(\mathrm{H}_{\alpha}\right)$.

Fig. 5. - Profils de répartition radiale de l'intensité totale de certaines raies et du fond continu correspondant $(F)$.

[Profiles of the radial distribution of the total intensity of some lines and the corresponding continuous spectrum F.]

de la colonne nécessaire pour pouvoir appliquer l'inversion d'Abel. Cette hypothèse est difficile à vérifier pour des raisons expérimentales et l'existence d'une symétrie selon l'axe $x$ ne constitue pas une preuve suffisante mais on peut ajouter les deux éléments suivants :

- l'observation de courbes obtenues dans les conditions citées précédemment mais en effectuant la visée suivant un axe perpendiculaire au précédent (perpendiculaire au plan de la figure 1, parallèlement aux barreaux d'isolants) montre qu'il n'y a aucune accumulation de vapeurs d'isolant près des échantillons;

- l'existence d'un vortex gazeux de stabilisation favorise une géométrie de révolution.

L'inversion des courbes de répartition de l'intensité absolue de la raie $415,8 \mathrm{~nm}$ de l'atome neutre d'argon permet de calculer la température du plasma.

3.2 LA TEMPÉRATURE. - Les courbes de répartition radiale de la température ont été tracées sur la figure 6 pour différentes valeurs de l'intensité de courant d'arc avec et sans contamination du plasma par des vapeurs d'amiante-ciment.

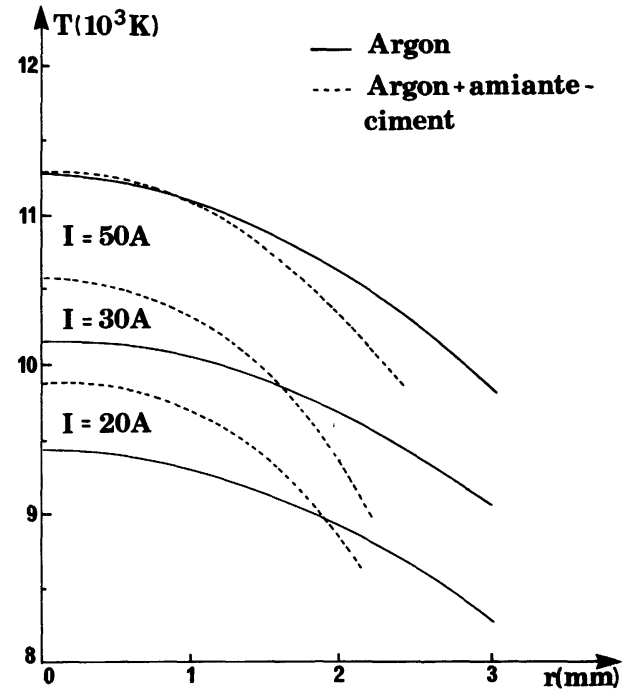

Fig. 6. - Répartition radiale de la température, pour trois valeurs de l'intensité du courant.

[Radial distribution of the temperature for three values of the current intensity.]

La valeur absolue de la température est déterminée avec une précision estimée à $5 \%$; les variations de température sont connues avec une précision de $1 \%$. La présence d'isolant :

- augmente la température sur l'axe de la décharge,

- augmente le gradient de température,

- diminue le rayon optique de l'arc.

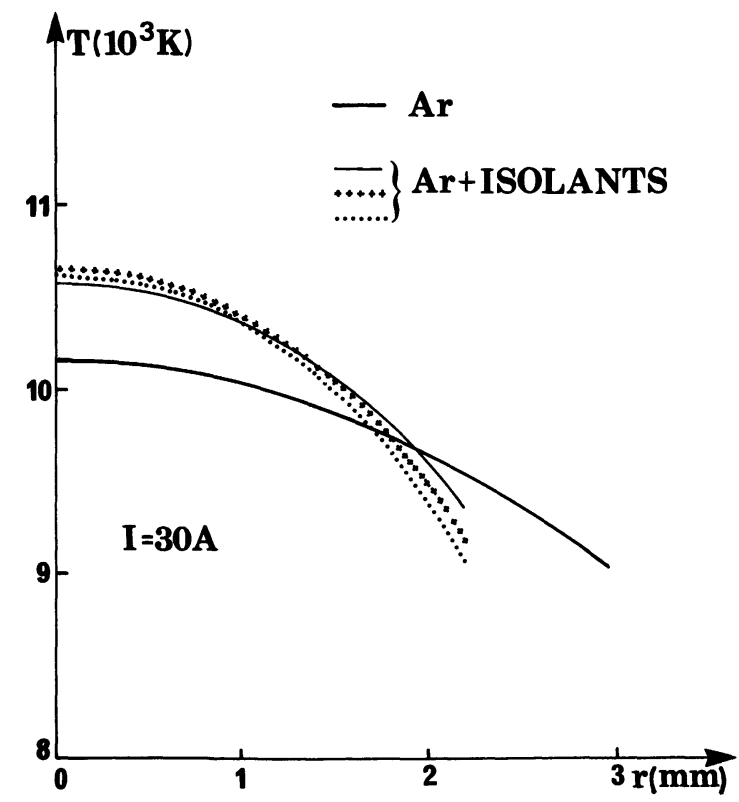

Fig. 7. - Répartition radiale de la température relative à chaque isolant : - amiante-ciment $;++$ amianteciment imprégné au silicone; ... tissu de fibre de verre imprégné de ciment.

[Radial distribution of temperature, according to each insulating material : - asbestos-cement $;++$ silicon abestos-cement ; ... glass-fibre reinforced with cement.] 
La faible augmentation de la température à $50 \mathrm{~A}$ est probablement liée à une auto-absorption de la raie d'argon. L'existence de ce phénomène n'a pas, pour le moment, été confirmée.

Sur les courbes de la figure 7 apparaît la répartition radiale de la température à $30 \mathrm{~A}$ lorsque le plasma est contaminé par des isolants de nature différente. On peut constater que tous les échantillons expérimentés conduisent à des résultats similaires permettant de confirmer le phénomène décrit précédemment.

3.3 LA DENSITÉ ÉLECTRONIQUE. - Le relevé des profils de la raie $\mathrm{H}_{\beta}$ de l'hydrogène provenant des isolants a été fait en deux points de la colonne, sur l'axe et près $\mathrm{du}$ bord. Les résultats obtenus à $40 \mathrm{~A}$ pour environ 50 essais montrent que la valeur moyenne de la densité électronique est de $3,6 \times 10^{16} \mathrm{~cm}^{-3}$ au centre et de $1,3 \times 10^{16} \mathrm{~cm}^{-3}$ sur les bords. Ces valeurs ne diffèrent pas sensiblement d'un isolant à l'autre. La comparaison de ces résultats avec ceux correspondant à un plasma d'argon pur (respectivement $3,4 \times 10^{16} \mathrm{~cm}^{-3}$ et $1,3 \times 10^{16} \mathrm{~cm}^{-3}$ déterminés à partir de la mesure de l'intensité du fond continu à $700 \mathrm{~nm}$ [6]) montre un léger accroissement de la densité électronique au centre de la décharge.

3. 4 LE CHAMP ÉLECTRIQUE. - L'évolution du champ électrique dans le temps a la forme décrite par la courbe de la figure 4. Nous retiendrons la valeur moyenne de la phase 3. Pour chaque mesure, on relève successivement la valeur du champ dans l'argon pur (phase 0) et dans l'argon contaminé par des vapeurs d'isolant (phase 3). Les résultats sont présentés dans le tableau I pour deux valeurs de l'intensité de courant d'arc. Les résultats présentés sont des moyennes correspondant à une dizaine de mesures. L'analyse de ces résultats montre que la présence de vapeurs d'isolants entraîne une augmentation de la valeur du champ électrique d'environ $20 \%$. Elle est de $30 \%$ dans le cas de la mélamine. Ceci laisse prévoir une variation de la conductivité électrique difficile à estimer à partir de ces résultats en raison de la variation du diamètre de la décharge.

Tableau I. - Le champ électrique.

[Electric field.]

\begin{tabular}{|c|c|c|c|c|}
\hline \multirow{2}{*}{ Plasma } & \multicolumn{2}{|c|}{$I=40 \mathrm{~A}$} & \multicolumn{2}{c|}{$I=50 \mathrm{~A}$} \\
\cline { 2 - 5 } & $E(\mathrm{~V} / \mathrm{m})$ & $\Delta E(\%)$ & $E(\mathrm{~V} / \mathrm{m})$ & $\Delta E(\%)$ \\
\hline $\begin{array}{c}\text { Argon } \\
\text { Argon-amiante } \\
\text { ciment }\end{array}$ & 490 & & 530 & \\
$\begin{array}{c}\text { Argon-ciment } \\
\text { fibre de verre }\end{array}$ & 573 & 21 & 662 & 25 \\
Argon mélamine & & 17 & 635 & 20 \\
\hline
\end{tabular}

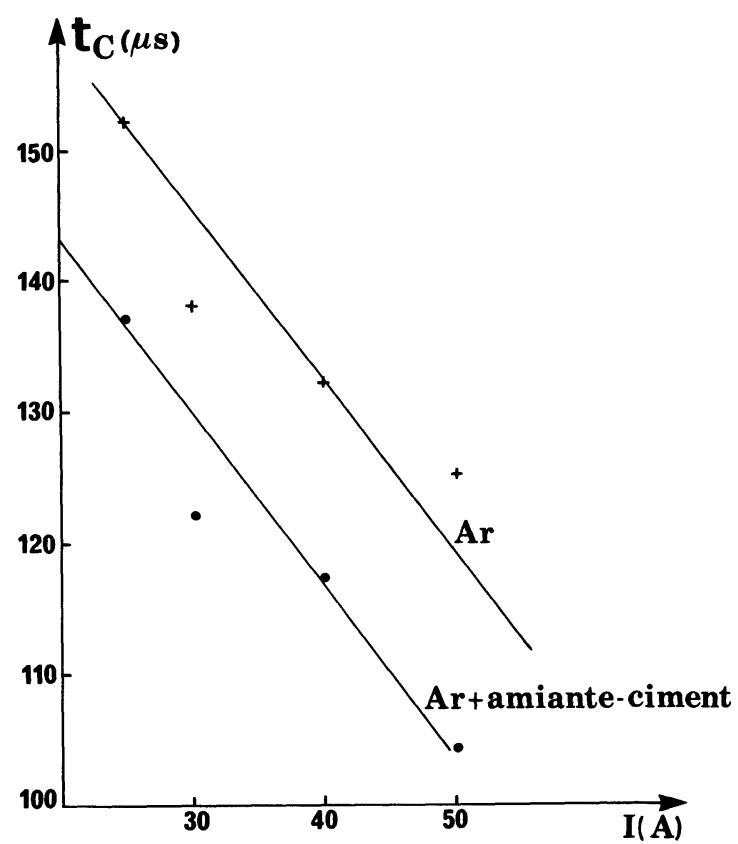

Fig. 8. - Variation du temps d'extinction en fonction de l'intensité du courant d'arc.

[Variation of the extinction time versus the intensity of the arc-current.]

3.5 La décharge en extinction. - Le temps d'extinction de l'arc $t_{\mathrm{c}}$ a été mesuré pour un plasma d'argon en présence et en l'absence de contamination par des vapeurs d'amiante-ciment pour différentes valeurs de l'intensité du courant d'arc (Fig. 8). Chaque point correspond à la moyenne d'environ 10 mesures. En présence de vapeurs d'isolant le temps d'extinction est diminué de 10 à $15 \%$. Aucune différence appréciable n'a pu être mise en évidence entre les isolants de différente nature.

3.6 ANAlYSE. - L'augmentation du gradient de température ainsi que la diminution du rayon optique de l'arc peuvent être liées à un accroissement de la conductivité thermique du plasma et/ou à un refroidissement beaucoup plus intense du jet en raison de l'énergie consommée par la vaporisation.

L'avancement des travaux n'est pas actuellement suffisant pour déterminer l'influence relative de la striction du jet de plasma par le refroidissement dû à l'évaporation et celle de la contamination de la décharge par des vapeurs d'isolants qui entraîne une modification des caractéristiques du plasma (composition, conductivités électrique et thermique). Mais, de nombreux essais faits avec des matériaux très divers, s'ils n'ont pas toujours conduit à des résultats exploitables, ont montré que, dans certains cas, il y avait vaporisation de l'isolant sans striction de l'arc. Il semblerait donc qu'une' première approche des arcs analysés au cours de cette étude puisse être faite en tenant compte de la modification de certaines grandeurs caractéristiques du plasma. 
Les résultats concernant le temps d'extinction de l'arc sont en bon accord avec les autres résultats présentés. En effet, la conductivité thermique d'un plasma augmente avec la température. L'évacuation de l'énergie interne de l'arc s'effectue d'autant plus facilement que la valeur de la température est plus élevée. Or, à une intensité de courant d'arc donnée, la température est plus élevée lorsque le plasma est contaminé par des vapeurs d'isolants. Ceci entraîne une diminution du temps d'extinction de l'arc. Hertz [7] a montré l'importance qu'occupe le transfert d'énergie de réaction dans la vitesse d'extinction de l'arc. Il a établi une relation liant la constante de temps de l'arc à la valeur maximale de la conductivité thermique de réaction $\left(\tau=\frac{\mathrm{Cte}}{\lambda_{\mathbf{R}}^{\max }}\right)$. Cette composante de la conductivité thermique est fortement augmentée par la présence dans le plasma de molécules à faible énergie de dissociation. De telles molécules entrent pour une large part dans la composition des isolants considérés. Il semblerait qu'il puisse exister également un mécanisme d'échange d'énergie interne entre particules [8].

\section{Conclusion.}

Les isolants industriels utilisés pour cette étude sont des matériaux qui permettent d'obtenir des chambres de coupure ayant un bon pouvoir de coupure. Malgré de nombreuses difficultés expérimentales liées aux instabilités inhérentes à une contamination qui reste malgré tout irrégulière, cette étude a permis de mettre en évidence que la présence dans la colonne d'arc de vapeurs d'isolants augmente la température, le champ électrique, la conductivité thermique et diminue le temps d'extinction de l'arc. Une recherche bibliographique a montré que très peu de travaux ont été publiés sur ce sujet. Si l'étude présentée a permis de montrer l'influence des vapeurs d'isolants sur certaines caractéristiques de la décharge et de prouver que la présence de ces vapeurs peut favoriser la coupure, d'autres travaux seront encore nécessaires pour expliquer les mécanismes mis en jeu.

\section{Remerciements.}

Ce travail a été réalisé sur contrat pour la Société Alsthom-Atlantique. Les auteurs remercient tout particulièrement $\mathrm{M}$. J. Ph. Gelez pour son aide et ses conseils.

\section{Bibliographie}

[1] Papadakis, M., Venuat, M., Laboratoire d'essais des ciments, mortiers, bétons (Eyrolles, Paris) 1969.

[2] Pascal, P., Traité de Chimie Minérale (Masson, Paris) 1965.

[3] Cheminat, B., Thèse de Doctorat ès-Sciences. No 311, Clermont-Ferrand (1983) France.

[4] Andanson, P., Cheminat, B., Halbique, A. M., J. Phvs. D 11 (1978) 209-15.
[5] Griem, H. R., Plasma Spectroscopy (McGraw-Hill New York) 1964.

[6] Rochon, J. P., 1982 Mémoire C.N.A.M. ClermontFerrand.

[7] Hertz, W., Z. Phys. 245 (1971) 105.

[8] Vacquie, S., Kafrouni, H., Dinguirard, J. P., J. Phys. D : Appl. Phys. 8 (1975) 191-200. 